(C) 2000 International Press

Adv. Theor. Math. Phys. 4 (2000) 617-626

\title{
Projective resolutions of
}

\section{coherent sheaves and descent}

\section{relations between branes}

\author{
A. Tomasiello \\ International School for Advanced Studies \\ via Beirut 2-4, 34014 Trieste, Italy \\ tomasiel@sissa.it
}

\begin{abstract}
We notice that, for branes wrapped on complex analytic subvarieties, the algebraic-geometric version of K-theory makes the identification between brane-antibrane pairs and lower-dimensional branes automatic. This is because coherent sheaves on the ambient variety represent gauge bundles on subvarieties, and they can be put in exact sequences (projective resolutions) with sheaves corresponding to vector bundles on the pair; this automatically gives a $D(p-2)$ as a formal difference of bundles on the $D p-D \bar{p}$ pair, both belonging to the Grothendieck group of coherent sheaves of the ambient.
\end{abstract}

e-print archive: http://xxx.lanl.gov/hep-th/9908009 


\section{Introduction}

There has been recently a major shift of perspective as to the interpretation of tachyons in brane-antibrane pairs [1]. It has been long since suspected that they signal instabilities, but one could not forecast whether this led to inconsistency of the theory or could have instead a more constructive role. It is now believed that the latter is the case, and that tachyons can be viewed simply as Higgs fields, whose condensation can lead to interesting effects. In particular, it can lead to remnants whose stability is in turn simply dictated by the topological stability of the condensation. Here we will be interested only in type II theories and in stable products of decay. If on the brane and on the antibrane there are gauge bundles $E$ and $F$ respectively, what we need is something that classifies the topological content of the pair. The key is to note that [2], if the two bundles are topologically the same, they will annihilate leaving no stable remnant: there is in this case no stable pattern of condensation for the tachyon. Moreover, if such a trivial pair is added to non trivial ones, it will disappear, giving no contribution. We can write this symbolically as $(E, F) \sim(E \oplus H, F \oplus H)$. Since this is the definition of topological $\mathrm{K}$-theory, we can interpret physics by identifying a brane-antibrane system as an element of this group (hints in this direction were explicitly given before in [3]).

We have now to identify physically the result of the decay, which in general is not the vacuum (as there exist non-trivial elements of $\mathrm{K}$ theory). The locus in which the tachyon vanishes is the place around which energy is located, and is a source for lower RR fields. So it is natural to identify it with a brane supported on its zero locus; this can be viewed as a section of $\mathcal{T} \equiv E \otimes F^{*}$. This second point makes it possible that K-theory also classifies all lower-dimensional D-branes.

Let us stress that the classification of pairs by K-theory and the identification of the remnant are, so far, two different steps. Note, indeed, that a K-theory class does not define the bundle $\mathcal{T}$ : if we change the representative, the latter changes as well.

Here we will argue that, if we deal with complex analytic subvarieties, the identification of the remnant is a natural consequence of the first step, in a way that puts a new light on the incorporation of lower charges. This is because in that case we can choose, instead of K-theory, 
an analytic (algebraic-geometric) version of it, the Grothendieck group of coherent sheaves $[4,5]$, hereafter referred to simply as Grothendieck group (as it is commonly meant in algebraic geometry; in general this name simply denotes the procedure to get a group from a monoid). In this setting, bundles over subvarieties are expressible - through projective resolutions - as formal differences of bundles over the ambient variety. This allows us to see the identification between brane-antibrane and K-theory on the same footing as that between brane-antibrane and lower-dimensional brane; in a way, this becomes automatic, or natural.

On the one hand, this seems to confirm the physical analysis already done; on the other, it allows us to interpret descent relations, and tachyon condensation between stable D-branes, in terms of projective resolutions. In turn, the physical identification between $D p-D \bar{p}$ and $D(p-2)$ can be viewed now as a purely topological counterpart of an algebraic-geometric procedure.

One should stress the difference between what we said above and the already known mathematical counterpart of tachyon condensation: the isomorphism between the K-theory of a submanifold and that of the ambient. While that is an isomorphism between different groups, what we propose here is that the descent relation can be viewed as natural in one single group. Even better, while that isomorphism is natural, it does not justify in itself the identification of the $D(p-2)$ with the pair. In our case, on the contrary, we treat complex analytic subvarieties and so we can choose to use Grothendieck groups: if we do that, the identification of the product of tachyon condensation is a logical consequence of the existence of projective resolutions of coherent sheaves. A related issue is the following. One often says that K-theory of the ambient space classifies branes: if so, not only should there be representatives in the group for all lower-dimensional branes, but also different classes for branes of different dimensions. While the first feature has been demonstrated to be there, the second would lack if we did not choose different boundary conditions (and thus compactifications of the ambient space, by adding points at infinity; for instance usually one takes spheres to classify branes on flat space), for example by considering higher K-theory groups. These groups together then form a ring which one considers as classifying branes. Our attitude here will be different: we will have everything in the same group, by taking, as compactification, complex projective space, which has the virtue of 
giving us a $\mathbb{Z}$ for every codimension. We will return to this later.

The following discussion can be fitted to any case in which we want to express a brane wrapped on a submanifold in terms of a pair wrapped on a manifold; just compactify the latter to a complex projective variety. The main case we have in mind is IIB theory, with space filling branes and antibranes; so in this case we take, as we said, projective space $\mathbb{P}^{5}$, of real dimension 10 , as ambient variety. The examples that we will be talking about in the following, however, are chosen to be easier. We do not make any claim of mathematical originality; these examples are here just to clarify and illustrate the general discussion, which also relies on well-known mathematical facts.

Issues about the role of Grothendieck groups were already considered in [6], with a different point of view and aim from the one we take here.

\section{Coherent sheaves and their projective resolutions}

The key concept we have to introduce, before discussing Grothendieck groups, is that of coherent sheaf. Sheaves generalize the concept of fibre bundles on a manifold, which can be viewed as a particular class of sheaves, the locally free ones. Fibre bundles on a submanifold will then be described precisely by coherent sheaves. On a projective smooth variety $X$, the case to which for simplicity we will now restrict, they are sheaves of finitely generated $\mathcal{O}_{X}$-modules, and thus (using, for example, regularity and the presence of an ample invertible sheaf) admit a finite projective free resolution, that is, they can be put in an exact sequence:

$$
0 \rightarrow \mathcal{F}_{n-1} \rightarrow \cdots \rightarrow \mathcal{F}_{1} \rightarrow \mathcal{F}_{0} \rightarrow \mathcal{C} \rightarrow 0
$$

where $\mathcal{C}$ is our coherent sheaf and the $\mathcal{F}_{k}$ are locally free. Fibre bundles on a closed subvariety are coherent sheaves (closed immersions are proper, and so take coherent sheaves in the subvariety - in particular, locally free ones - to coherent sheaves), and so admit such resolutions; we will see how this works in examples. Note that our conclusions are untouched by possible singularities of the subvarieties. 
There are in general two definitions of Grothendieck groups. Take $\mathbb{Z}[\mathcal{L}]$ (respectively $\mathbb{Z}[\mathcal{C}]$ ) the free abelian group generated by locally free (respectively coherent) sheaves. Then

$$
\mathcal{K}^{\bullet} \equiv \mathbb{Z}[\mathcal{L}] / \mathcal{I}, \quad \mathcal{K}_{\bullet} \equiv \mathbb{Z}[\mathcal{C}] / \mathcal{I}^{\prime},
$$

where $\mathcal{I}\left(\mathcal{I}^{\prime}\right)$ is the ideal generated in both cases by elements of the form $\mathcal{F}-\mathcal{F}^{\prime}-\mathcal{F}^{\prime \prime}$ if there is an exact sequence $0 \rightarrow \mathcal{F}^{\prime \prime} \rightarrow \mathcal{F} \rightarrow \mathcal{F}^{\prime} \rightarrow 0$. In the case of smooth projective varieties, these two definitions are the same (the two groups are isomorphic). In particular we can immediately see that every coherent sheaf can be expressed as an element of $\mathcal{K}^{\bullet}$, by their defining sequence $(1)$, and so as the difference $\mathcal{E}-\mathcal{F}$ of two locally free sheaves. This simply means that in this setting we can express a fibre bundle on a subvariety as the difference of two fibre bundles on the variety. This is just the identification given by tachyon condensation! So we see that, if we use Grothendieck group instead of topological K-theory, the first step we described above (the fact that we can cancel the same bundle if it appears on both sides) implies the second (tachyon condensation and identification with lower-dimensional branes). Of course we have not shown the isomorphism between Grothendieck groups here: but this point is also essential, because otherwise the class which we found would depend on the projective resolution we chose. Although we will not prove that here [5], we will check it explicitly in an example at the end of the paper.

This group $\mathcal{K}^{\bullet} \cong \mathcal{K}_{\bullet}$ is different from $\mathrm{K}$-theory in various respects. First, of course, we are working with holomorphic subvarieties, and choosing holomorphic structures for the bundles involved. Second, what we identify with zero are extensions instead of direct sums. But all extensions are topologically trivial (direct sums), so this is not a problem. So this group does not contain in general all of what is contained in K-theory (the non-complex bundles); this gives us a means to understand which brane-antibrane configurations represent lower dimensional branes wrapped on holomorphic cycles. At the same time this group distinguishes objects which in K-theory are the same.

We have still to verify that the isomorphism between the Grothendieck groups is really consistent with the tachyon condensation we already know. We will do this in several examples, using as a basic tool the sequence

$$
0 \rightarrow \mathcal{F} \rightarrow \mathcal{F}(D) \rightarrow \mathcal{F}(D)_{\left.\right|_{D}} \rightarrow 0
$$


with $\mathcal{F}$ locally free (alternatively, we can derive it from (3) tensoring). Start from a brane wrapped on a holomorphic cycle with its bundle - that is, a coherent sheaf. We have to express it in terms of locally free sheaves (brane-antibrane) and then do tachyon condensation to see whether we retrieve the initial configuration. Let us start with the case of one brane, with the structure sheaf as bundle on it (we think of the $U(1)$-bundle as the circle bundle inside it), and suppose that we work in $X=\mathbb{P}^{n}$. The resolution depends on whether the subvariety $Y$ is a complete intersection or not; in the former case it is easy. In the case of (real) codimension 2 it reads

$$
0 \rightarrow \mathcal{O}_{X}(-Y) \rightarrow \mathcal{O}_{X} \rightarrow \mathcal{O}_{Y} \rightarrow 0
$$

the first term is the ideal sheaf, that we can think as the sheaf of holomorphic functions having a zero along $Y$. From this we get that $\mathcal{O}_{Y}=$ $\mathcal{O}_{X}-\mathcal{O}_{X}(-Y)$, and so the tachyon is a section of $\mathcal{E} \otimes \mathcal{F}^{*}=\mathcal{O}_{X}(Y)$, hence it has a simple zero on $Y$ (recall there is a duality between functions having poles and sections having zeroes). This exactly parallels the already known construction of a brane-antibrane pair which reproduces a codimension 2 brane. We already remarked that the bundle of which the tachyon is a section is not defined by the class in $\mathrm{K}$-theory; instead, it is guessed in some way by thinking about a "minimal" couple $(E, F)$. With this procedure, this minimality is just the concept of minimal resolution of a coherent sheaf, well-known to algebraic geometers.

\section{Higher codimension.}

Let us describe what to do if the codimension is higher, sticking with the case of complex projective spaces. Exploiting the fact that line bundles, in this case, are classified by $\mathbb{Z}$, we only write degrees. Let $d_{1}, \ldots, d_{l}$ be the degrees of the equations $f_{1}, \ldots, f_{l}$ defining the subvariety, and suppose it to be a complete intersection,

$$
\begin{aligned}
0 \rightarrow \mathcal{O}_{X}\left(-d_{1}-\cdots-d_{l}\right) \rightarrow \cdots & \rightarrow \bigoplus_{i_{1}, i_{2}} \mathcal{O}_{X}\left(-d_{i_{1}}-d_{i_{2}}\right) \\
& \rightarrow \bigoplus_{i} \mathcal{O}_{X}\left(-d_{i}\right) \stackrel{\phi}{\rightarrow} \mathcal{O}_{X} \rightarrow \mathcal{O}_{Y} \rightarrow 0
\end{aligned}
$$

The origin of this resolution can be understood as follows: the $\mathcal{V} \equiv$ $\oplus_{i} \mathcal{O}\left(-d_{i}\right)$ term is the generalization of the first (from the left) term 
of (3) due to the fact that we have more than one equation, and may be thought as the sheaf of $l$-uples of functions $\left(f_{1} h_{1}, \ldots, f_{l} h_{l}\right) ; \phi$ takes such an $l$-uple to $\sum f_{i} h_{i}$, and the next term on the left is its kernel, i.e., relations between the $f_{i}$. We always find the obvious relations $f_{i} \cdot f_{j}-f_{j} \cdot f_{i}=0$, which generate the term $\oplus_{i, j} \mathcal{O}\left(-d_{1}-d_{j}\right)$; in the case of non complete intersections, the number of equations necessary to define the variety would be higher than the codimension, and we would have extra terms. In fact in the complete intersection case one may make (4) more transparent in the form

$$
0 \rightarrow \Lambda^{l} \mathcal{V} \rightarrow \cdots \rightarrow \Lambda^{2} \mathcal{V} \rightarrow \Lambda \mathcal{V} \rightarrow \mathcal{O}_{X} \rightarrow \mathcal{O}_{Y} \rightarrow 0
$$

also known as the Koszul complex. Note that we do not have to proceed step by step: this is an "all at once" procedure, as the one already known and involving $\mathrm{K}$-theory Thom isomorphism and ABS construction ([2]; see also [7] for a more extensive description). To see better the correspondence, first remember that for any complex

$$
E_{0} \stackrel{d_{0}}{\rightarrow} E_{1} \stackrel{d_{1}}{\rightarrow} \cdots \stackrel{d_{n-1}}{\rightarrow} E_{n}
$$

one may construct a smaller complex (a map)

$$
\bigoplus_{i=\text { even }} E_{i} \stackrel{D}{\rightarrow} \bigoplus_{i=\text { odd }} E_{i}
$$

where $D\left(e_{0}, e_{2}, \ldots\right)=\left(d_{0} e_{0}+d_{1}^{*} e_{2}, d_{2} e_{2}+d_{3}^{*} e_{4}, \ldots\right)$; moreover the latter complex is exact if the former is. If we apply this to the sequence (4), then we have a pair of bundles which just correspond to the Clifford bundles $\left(\mathcal{S}_{+}, S_{-}\right)$associated to the conormal of our variety; for example Clifford multiplication (which can be viewed as a sum of an exterior product and a contraction) arises from the sum in the definition of $D$.

Projective resolutions like this are also known, when thought in terms of the corresponding algebraic objects (rings and modules) as syzygies, [8].

Lower charges.

We will now give more concrete examples for the case of a non trivial line bundle on the $(p-2)$-brane. Since we will do this again in projective spaces, let us return for a moment to the point we anticipated in 
the introduction. One would imagine, from the descriptions we gave so far of the identifications of the $D p-D \bar{p}$ system with lower dimensional branes, that, fixed our ambient space, K-theory or its Grothendieck group already provides a classification of all branes of all dimensions. To check this we have to choose the space; even if we have fixed the dimension $p$, we still have to choose the boundary conditions. This amounts to choosing a type of compactification. We can choose, as usual, a sphere; but the K-theory of a sphere is at most $\mathbb{Z}$. This means that the construction, by itself, would confuse branes of different dimensions: two dimensionally different branes can be realized as the same $D p-D \bar{p}$ system. We have, so to say, to avoid this by hand, treating each codimension separately and imposing that our bundles depend just on the transversal dimensions. If we do not do that, we find that in a given brane-antibrane system, a $(p-2)$ brane is given by a certain couple $(E, F)$, which is anyway also topologically the same as a couple which gives a $(p-4)$ brane.

We point out that it could be that different choices of boundary conditions (that is, of compactification) can give a better result. For instance, choosing the complex projective space $\mathbb{P}^{n}$, both K-theory and the Grothendieck group are isomorphic to $[5,9]$

$$
R \equiv \mathbb{Z}[t] /(t-1)^{n+1},
$$

which gives a $\mathbb{Z}$ for each even codimensional brane (the isomorphism from $\mathcal{K}_{\bullet}$ or $\mathrm{K}$-theory to $R$ is given by sending the hyperplane $H$ to $t$ ). The fact that the topological and algebraic geometrical K-theory are equal here is reasonable, since we know that $\mathbb{P}^{n}$ has no Jacobian. In a way the trick is that, choosing $\mathbb{P}^{n}$ as compactification space, we allowed all possible boundary conditions, so distinguishing between branes of different dimensions. Of course in this way we are not predicting the allowed codimensions of the branes: we cannot because we chose the complex analytic setting, forcing them to be even.

Now we return to the examples we wanted to give: non trivial line bundles on the $(p-2)$ brane. This is easier if we can extend the line bundle to one on the ambient space, whose divisor let us call $D$ : indeed, applying again (2), we obtain

$$
0 \rightarrow \mathcal{O}_{X}(D-Y) \rightarrow \mathcal{O}_{X}(D) \rightarrow \mathcal{O}(D)_{\left.\right|_{Y}} \rightarrow 0
$$

the last term is just our original bundle on the subvariety. Note that again $\mathcal{E} \otimes \mathcal{F}^{*}=\mathcal{O}_{X}(Y)$, as it should. To fix ideas, consider the case of 
a conic in $\mathbb{P}^{2}$, which is birationally equivalent to $\mathbb{P}^{1}$. If the line bundle is $\mathcal{O}_{\mathbb{P}^{1}}(2)$, it can be extended on $\mathbb{P}^{2}$ as $\mathcal{O}(1)$ (henceforth $\mathcal{O} \equiv \mathcal{O}_{\mathbb{P}^{2}}$ ); the sequence so reads

$$
0 \rightarrow \mathcal{O}(-1) \rightarrow \mathcal{O}(1) \rightarrow \mathcal{O}_{\mathbb{P}^{1}}(2) \rightarrow 0
$$

Life is harder if we consider instead the bundle $\mathcal{O}_{\mathbb{P}^{1}}(1)$, which is not extendable to $\mathbb{P}^{2}$, since there is no divisor on it which intersects the conic once. We can circumvent this difficulty in two ways. First, we can start the projective resolution from $\mathcal{O} \oplus \mathcal{O}$, instead of from $\mathcal{O}$. Doing this carefully involves looking at the rings of functions over the varieties, and maps between them; we will not delve into details, but the result turns out to be

$$
0 \rightarrow 2 \mathcal{O}(-1) \rightarrow 2 \mathcal{O} \rightarrow \mathcal{O}_{\mathbb{P}^{1}}(1) \rightarrow 0
$$

(we denote now $\oplus_{1}^{n} \mathcal{O}(k)$ as $n \mathcal{O}(k)$ ). As a check, let us do it in another way, which only involves sequences that we have already seen. Write first the two sequences

$$
\begin{gathered}
0 \rightarrow \mathcal{O}_{\mathbb{P}^{1}}(-1) \rightarrow \mathcal{O}_{\mathbb{P}^{1}} \rightarrow \mathcal{O}_{p} \rightarrow 0 \\
0 \rightarrow \mathcal{O}(-2) \rightarrow 2 \mathcal{O}(-1) \rightarrow \mathcal{O} \rightarrow \mathcal{O}_{p} \rightarrow 0
\end{gathered}
$$

where $p$ is a point in $\mathbb{P}^{1}$ at which a section of our line bundle $\mathcal{O}_{\mathbb{P}^{1}}(1)$ vanish. Now, tensor both with an hyperplane line bundle in $\mathbb{P}^{2}$ : we get

$$
\begin{aligned}
\mathcal{O}_{\mathbb{P}^{1}}(1)=\mathcal{O}_{\mathbb{P}^{1}}(2)-\mathcal{O}_{p}(1) & =\mathcal{O}(1)-\mathcal{O}(-1)-(\mathcal{O}(1)-2 \mathcal{O}+\mathcal{O}(-1)) \\
& =2 \mathcal{O}-2 \mathcal{O}(-1)
\end{aligned}
$$

as above. Still another method would have been tensoring the first sequence in (6) by an hyperplane line bundle of $\mathbb{P}^{1}$, and then reexpressing $\mathcal{O}(1)$ as $3 \mathcal{O}-3 \mathcal{O}(-1)+\mathcal{O}(-2)$ : this gives an illustration of the relation $(t-1)^{n+1}=0$ in (5). The result is in any case reasonable because a conic can be deformed to two hyperplanes touching in a point.

If finally we want to consider stacked branes, $U(1)$-bundles become $U(r)$-bundles. So the generalization is to represent $U(r)$ on $\mathbb{C}^{r}$; we get in this way a complex vector bundle of rank $r$ on the lower-dimensional brane. The coherent sheaf is now again a sheaf of $\mathcal{O}$-modules, but with more generators; and so the resolution starts with $r \mathcal{O}_{X}$ instead of with $\mathcal{O}_{X}$. This is right, since to get stacked branes we expect to have to start 
from stacked brane-antibrane pair. The procedure works the same way, but to verify that we get the desired subvariety we have to look at the locus where the tachyon is non invertible, rather than zero.

We thank L. Bonora, A. Sen, A. Zampa for useful discussions and advice.

\section{References}

[1] A. Sen, SO(32) Spinors of Type I and other Solitons on BraneAntibrane Pair, J. High Energy Phys., 9809 (1998), 023, hep-th/9808141; Tachyon Condensation on the Brane-Antibrane System, J. High Energy Phys., 9808 (1998), 012, hep-th/9805170.

[2] E. Witten, D-Branes and K-Theory, J. High Energy Phys., 9812 (1998), 019, hep-th/9810188.

[3] R. Minasian and G. Moore, K-Theory and Ramond-Ramond Charge, J. High Energy Phys., 9711 (1997), 002, hep-th/9710230.

[4] R. Hartshorne, Algebraic Geometry, Springer-Verlag, New York, 1977.

[5] Yu.I. Manin, Lectures on the K-functor in algebraic geometry, Russ. Math. Surveys, 24 (1969), 1-89.

[6] E. Sharpe, D-Branes, Derived Categories and Grothendieck Groups, hep-th/9902116.

[7] K. Olsen and R.J. Szabo, Constructing D-branes from K-theory, hep-th/9907140.

[8] J.Harris, Algebraic Geometry: a first course, Springer-Verlag, New York, 1992.

[9] M.F. Atiyah, K-Theory, Benjamin, 1964. 